\title{
Construction and Optical Testing of Inflatable Membrane Mirror Using Structured Light Technique
}

\author{
Felipe Patiño-Jiménez, ${ }^{1}$ Yuri Nahmad-Molinari, ${ }^{1}$ Víctor Iván Moreno-Oliva, ${ }^{2}$ \\ Fátima De Los Santos-García, ${ }^{1}$ and Agustin Santiago-Alvarado ${ }^{3}$ \\ ${ }^{1}$ Instituto de Física, Universidad Autónoma de San Luís Potosí, Manuel Nava No. 6, Zona Universitaria, 78290 San Luis Potosí, \\ SLP, Mexico \\ ${ }^{2}$ Universidad del Istmo, Campus Tehuantepec, Ciudad Universitaria s/n, Barrio Santa Cruz, $4 a$ Sección, \\ 70760 Santo Domingo Tehuantepec, OAX, Mexico \\ ${ }^{3}$ Universidad Tecnológica de la Mixteca, Carretera Acatlima Km 2.5, 69000 Huajuapan de León, OAX, Mexico
}

Correspondence should be addressed to Felipe Patiño-Jiménez; fpatino@uteq.edu.mx

Received 5 February 2015; Revised 22 April 2015; Accepted 9 June 2015

Academic Editor: Yong Shuai

Copyright (C) 2015 Felipe Patiño-Jiménez et al. This is an open access article distributed under the Creative Commons Attribution License, which permits unrestricted use, distribution, and reproduction in any medium, provided the original work is properly cited.

\begin{abstract}
Construction and characterization of an inflatable mirror prototype made out of flexible polymeric membranes are being presented. Surfaces were curved by imposing a slight excess of air pressure. Lightweighted, lowcost, and commercially available materials were selected in order to produce solar concentration elements at competitive prices. In this sense, large-area, image-forming mirrors with low optical acuity were achieved by concentration purposes. Optical characterization of the mirror's shape at a given pressure or curvature radius was done by means of a structuredlight technique with a resolution of $0.1 \mathrm{~mm}$ finding a conical shape acquired by the inflated mirror as the best approximation. Concentration ratio achieved for a focal length of $5068 \mathrm{~mm}$ was of 25.1 suns, making a promising approach for lowering initial investment costs in applications such as hot-water, parabolic dish with Stirling engines, or concentrated photovoltaic electricity generation.
\end{abstract}

\section{Introduction}

Manufacturing an inflatable mirror requires two thin circular membranes that are sealed at their periphery and secured in a tension ring and injecting a certain amount of pressurized air between them sufficient to produce the focal ratio required for the mirror [1]. Inflatable mirrors have been used for various applications where low image sharpness is sufficient, such as microwave antennas and solar energy concentrators, not being used for optical imaging, where high acuity is required. Due to the fact that the profile of the inflated membrane is neither spherical nor parabolic, but instead an oblate spheroid, which is expressed in terms of a power series, called Hencky curve [1], this oblateness produces a spherical aberration, and a secondary optic element would be needed in order to compensate for such aberration resulting in a Cassegrain configuration.
By developing a thickness variable membrane theory [1], it was found that a radial thickness variation or a parabolic thickness profile of the membrane corrects the Hencky effect to achieve the shape of the inflated mirror, a paraboloid, in further studies on variable thickness membranes for inflatable mirrors [2], showed that the RMS error [3] of deployable optics can be reduced to allow image formation in visible bands and by limiting the effective optical surface area it can be used as a primary mirror in the infrared bands for astronomical telescopes.

Nowadays there is growing interest in the construction and study of concentration systems of solar radiation for thermal or electric energy production. Based on Bishop and Rossow's solar irradiance map, theoretically there are six collecting points of $151 \mathrm{~km}^{2}$ on average each one would be enough to generate the total primary energy supply used by humankind $[4,5]$. 


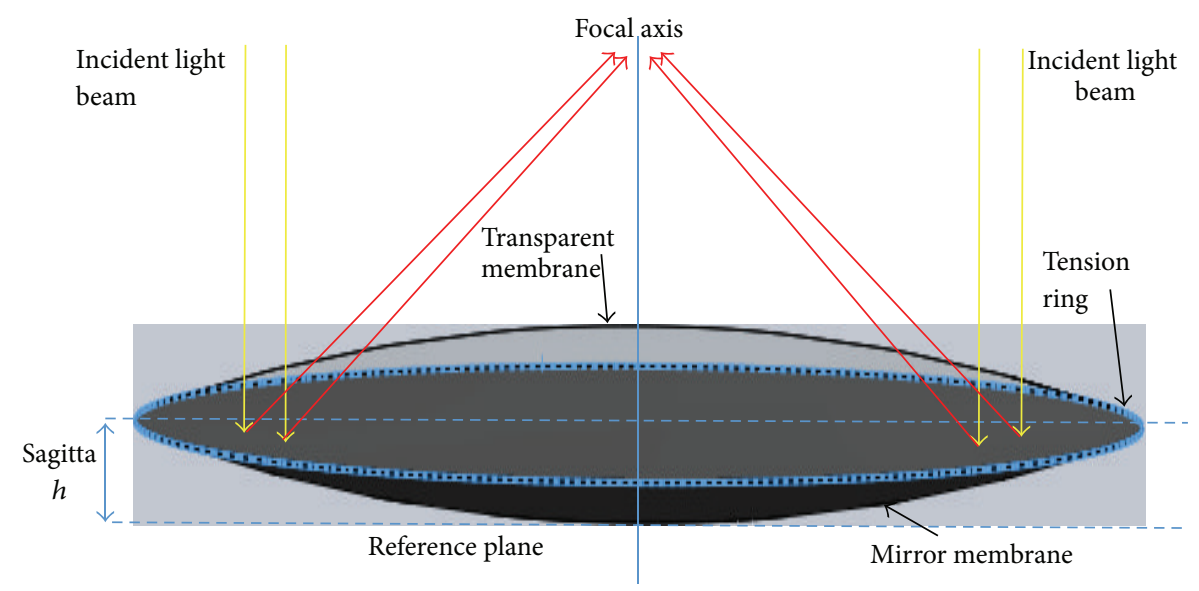

FIGURE 1: Outline/sketch inflatable membrane mirror.

Various point-like, line, and unfocused geometries have been used for concentration of solar radiation [6]. Particularly, solar parabolic dish concentrators offer the highest thermal and optical efficiency compared with all of solar concentrators demonstrated to date and allow higher concentration ratios, with a theoretical limit of 46,000 for an ideal specular reflecting surface [7]; however, they have much higher costs per unit area compared to linear systems [8], costs that rapidly increase with increasing reflector quality [9].

A solar concentrator constructed with inexpensive mirror facets and light-weighted structure can be a lower cost alternative to be used in concentrated solar thermal power applications. This is because current technologies use heavy special steel structures and expensive glass mirrors, not commercially available for smaller-scale applications.

Inflatable circular mirrors with pneumatically curved surface, supported on light structures, and built with flexible polymer membranes could be a low cost alternative to use in concentrated solar power. One of such mirrors is sketched in Figure 1, which corresponds to our design.

A few decades ago, aluminized polyester films have being attractive for manufacturing mirrors because of their high optical quality and low cost [9]; but cost saving in solar concentrators requires research, design, and development in areas such as evaluation and selection of materials, coupling membranes, and design of the supporting structure [10].

Eagerly trying to cut costs in concentration systems of solar radiation, faceted arrangements have been developed. In this way, a model of truncated parabolic dish with 164 facets was built, with an average concentration ratio of 108 suns, which could produce $8.15 \mathrm{~kW}$ heat in the focal plane which was built [11]. Another model of solar concentrator was made of ellipsoidal aluminized polyester mirrors membrane facets distributed on a paraboloid structure. In this last study they reached high values of solar concentration on the order of 8199 suns, reporting efficiencies in electricity production of $25 \%$ and higher [12]. In the latter technique used to make mirrors, they use polyester membranes prestressed and clamped between two elliptical rings that afterwards were curved into the shape of a mold by vacuum negative pressure application. On space applications, a photovoltaic array or the high-temperature end of a dynamic engine is other examples for inflatable membrane mirror application [13].

\section{Mirror Manufacturing}

In order to construct the concentrating mirror, flexible polymeric membranes of high reflectance and high transmittance were used as well as commercially available materials and tools, being primarily metallic polyester commercially known as Mylar, transparent polycarbonate, and galvanized metal pipe.

Through the process of cold rolling, a $3000 \mathrm{~mm}$ in length and $12.52 \mathrm{~mm}$ in diameter conduit pipe is bent into the form of a ring of $820 \mathrm{~mm}$ in outside diameter. After the excess is cut the inner ends were mechanically jointed through copper tubing $12.52 \mathrm{~mm}$ in diameter and $50 \mathrm{~mm}$ in length. This process can be seen in Figure 2(b). Ray tracing free software SolTrace [14] was used for functional simulation of the mirror and concentration ratio evaluation, Figure 2.

Unlike the design technique used to construct mirrors with curved polyester membranes using vacuum pressure [12], in this work the membrane becomes curved by positive pressure application. In both approaches a closed environment is required. Two circles of $830 \mathrm{~mm}$ diameter, one of $0.05 \mathrm{~mm}$ thick Mylar film and one of polycarbonate $0.15 \mathrm{~mm}$ thick, are placed to form a sandwich, leaving the metallic coating inside. A curing adhesive film with UV light is applied at the edges for bonding both membranes. After that, we performed a thermal cutting in order to eliminate a ring of $5 \mathrm{~mm}$ wide along the perimeter. This procedure also helps to thermally bind both membranes, sealing them together.

In order to assembly the mirror, first the membranes are mounted on the metal ring by a set of twelve equally distributed or equidistant points along the circumference of the ring; then with low-adhesion tape the sandwich membrane is stuck, alternating diametrically opposite points and applying slight tension to avoid deformation. Having done this, we proceeded with a high-adhesion metal tape 


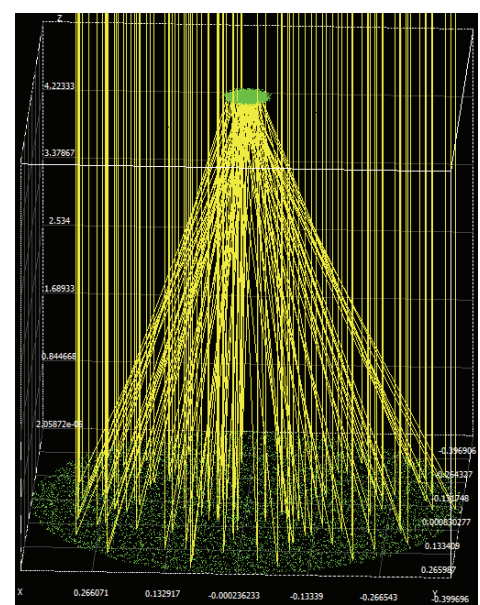

(a)

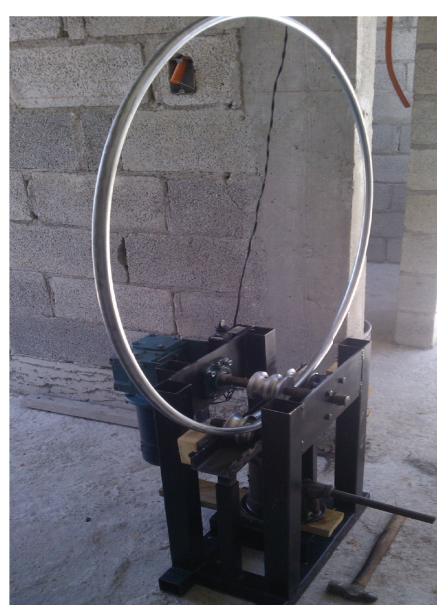

(b)

Figure 2: (a) Functional simulation by ray tracing. (b) Metal ring in the manufacturing process.

to fix the entire perimeter of the membranes on the ring; a higher tension in each section that covers the tape must be applied in order to do so.

Air was injected through a valve placed in between both membranes at low pressure up to $48 \mathrm{~Pa}$, in order to reach the required radius of curvature. The radius was monitored by measuring the sagitta or the height $h$ of the metallized membrane, as measured from the level of the edge of the ring.

\section{Proposed Method for Optical Testing of the Mirror}

Having manufactured the prototype, and once checked for perfect sealing, we proceeded to perform the optical characterization of the lenticular geometry of our mirror. For this, we used a geometric optical test based on optical triangulation with structured light [5]. It consists in projecting a line of laser light on the metalized surface of the membrane mirror; the laser beam is projected to an angle $\alpha$ relative to the horizontal plane ( $X-Y$ plane) and a charge-coupled device (CCD) camera of $15 \mathrm{Mpx}$ observes the projection of the line on the reflecting surface from above (Figure 3 ). The intersection between the laser beams with a horizontal plane would define a line. However, as the air contained within the sandwiching membranes rounds up the reflecting surface, the intersection of these surfaces with the laser beam gets distorted. The deviation from the ideal line defined by two intersecting planes is proportional to the local elevation of the surface with respect to a horizontal plane of reference passing through the borders of the supporting ring [6].

These elevation changes are observed in the image plane at the CCD camera as lateral displacements $\delta x$ (measured in pixels). The line that makes contact with the surface is distorted due to changes in height $\Delta z$ above the reference plane.

The expression of these lateral displacements associated with changes in height $\Delta z$ (in $\mathrm{mm}$ ) is determined by

$$
\Delta z=\frac{b \delta x \tan \alpha}{a+\delta x \tan \alpha},
$$

where $a, b$, and $\alpha$ are parameters depending on the optical system. The parameter $a$ is the distance between the diaphragm pinhole and the plane of the camera sensor, $b$ is the distance between the diaphragm pinhole and the reference plane, $\delta x$ is the lateral displacement of the laser line in the image plane of the camera, and $\alpha$ is the angle at which the laser line is projected. The projection is performed on the radius of the mirror at the reference plane and for a complete reconstruction of the mirror surface an angular swept was performed, rotating the mirror about its optical axis.

The parameter $a$ is determined using the lens equation by means of approximation:

$$
a \approx \frac{f *(c c d y+D)}{D},
$$

where $D$ is the diameter of test mirror. The resolution at which the lateral displacements are measured is determined by the minimum displacement that can be observed by the camera, which is about one pixel.

The parameters for the evaluation mirror surface are presented in Table 1.

The expression used to determinate the resolution is $(1)$, where $\delta x(\mathrm{~mm})=[(1$ pixel $)($ sensor size $(\mathrm{mm}))] /$ sensor size (pixels).

A direct compute result in a resolution of $0.17 \mathrm{~mm}$ with the parameter used in the test. See Table 1.

\section{Experimentation Testing of the Mirror}

The experimental determination of the shape of the mirror surface was performed by mounting an optical triangulation setup, consisting in a laser sheet projection system using a solid-state laser $(0.9 \mathrm{~mW}, 635 \mathrm{~nm})$ with a cylindrical lens; a double-membrane inflatable mirror as the testing object; and a CCD camera (Canon DS126291) for image capture and their posterior analysis. The mirror is mounted onto a rotating base driven by a stepper motor (Applied Motion HT34-506DYAA). 


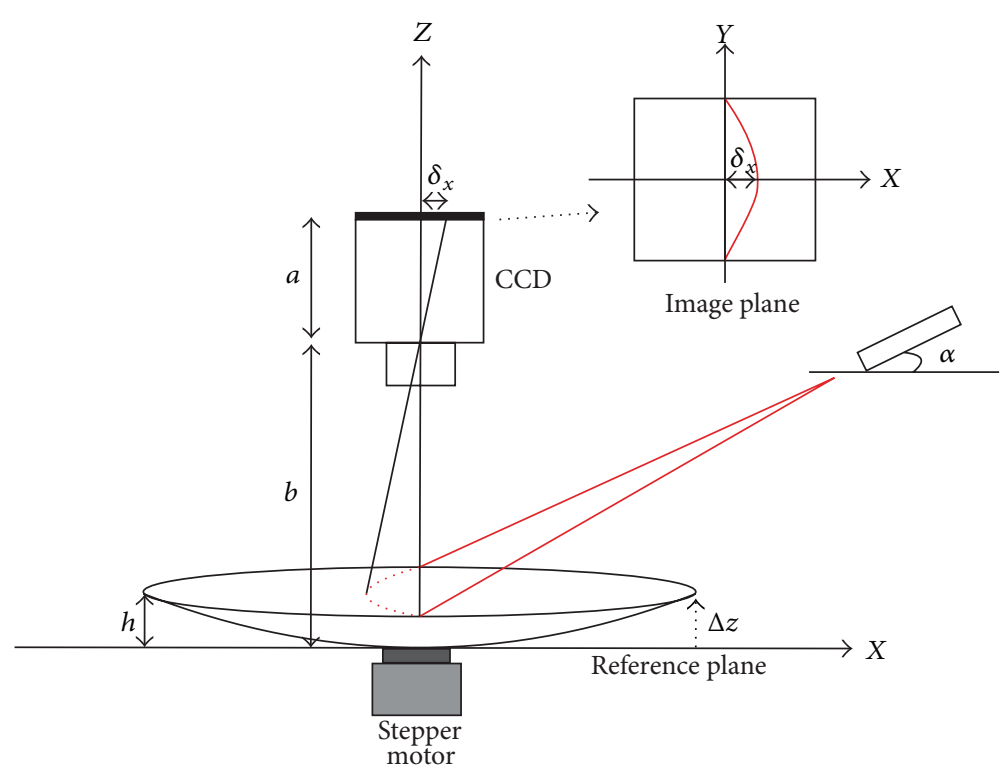

Figure 3: Experimental model setup for testing mirror's geometry.

TABLE 1: Parameters used in the mirror test.

\begin{tabular}{lc}
\hline Parameters & Value \\
\hline$f$ & $18 \mathrm{~mm}$ \\
$A$ & $18.64 \mathrm{~mm}$ \\
$B$ & $522.73 \mathrm{~mm}$ \\
$A$ & $28.64^{\circ}$ \\
Sensor size & $22.2 \times 14.8 \mathrm{~mm}$ \\
$D$ & $(1920 \times 1280$ pixels $)$ \\
\hline
\end{tabular}

The laser line is projected on the half-mirror surface of the membranes at an angle of inclination $\alpha$. The camera captures an image and the distorted line by changes in height in the region of the surface is measured in pixels by the camera. Hence, (2) determines the coordinate $z$ of the point on the surface. These lateral motions are measured pixel by pixel on the captured image that is along the line image on the sensor ( $y$-axis). For full reconstruction, 200 steps per revolution took place $\left(1.8^{\circ}\right.$ per step); that is, 200 images were captured and processed. The experimental arrangement is shown in Figure 4.

We carried out the evaluation process in a dark room isolated from outside stray light, so that the image processing can be performed under the same threshold value. Figure 5 shows the image where multiple reflections of the laser line are clearly observed. The line labeled "a" corresponds to the first reflection of the laser line impinging on the transparent polycarbonate layer. Line " $b$ " corresponds to the line reflected on the Mylar surface and is the one considered in this work in order to determine the mirror's shape. Finally, line " $c$ " is the reflection of line " $a$ " on the Mylar surface. The beam projected onto the metallized surface is the central beam "b." This is relevant to the processing of each image.

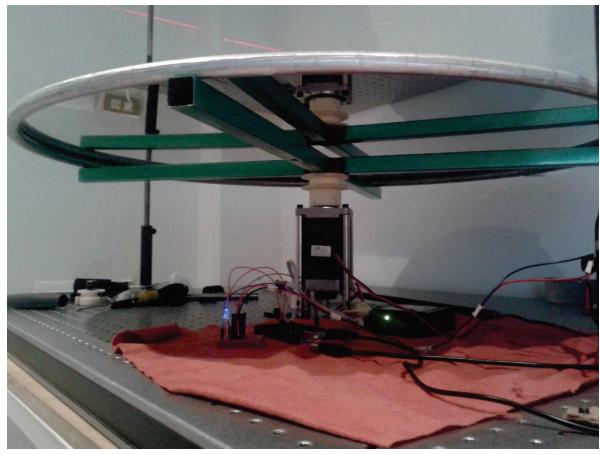

FIGURE 4: Experimental setup for evaluating the flexible membrane mirror.

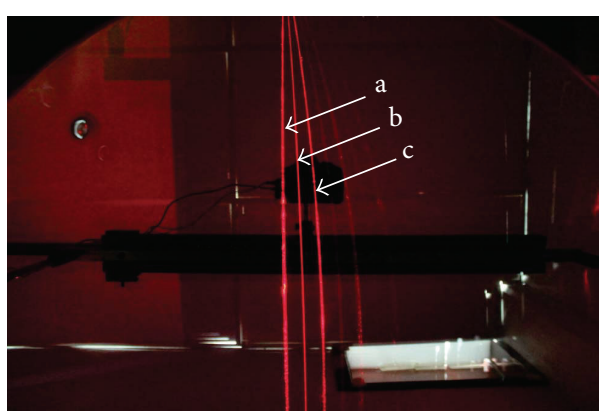

FIGURE 5: Projection of the laser line on the membranes of the mirror, (a) line incident on the transparent polycarbonate membrane, (b) line incident on the Mylar membrane, and (c) line reflected by the metallic surface.

4.1. Image Processes. Each resulting image was independently processed by means of a developed program on MATLAB software, in order to determine the intensity centroids for each light line. For doing so, the image was converted to grayscale, using the "rgbgray" feature and smoothed by 


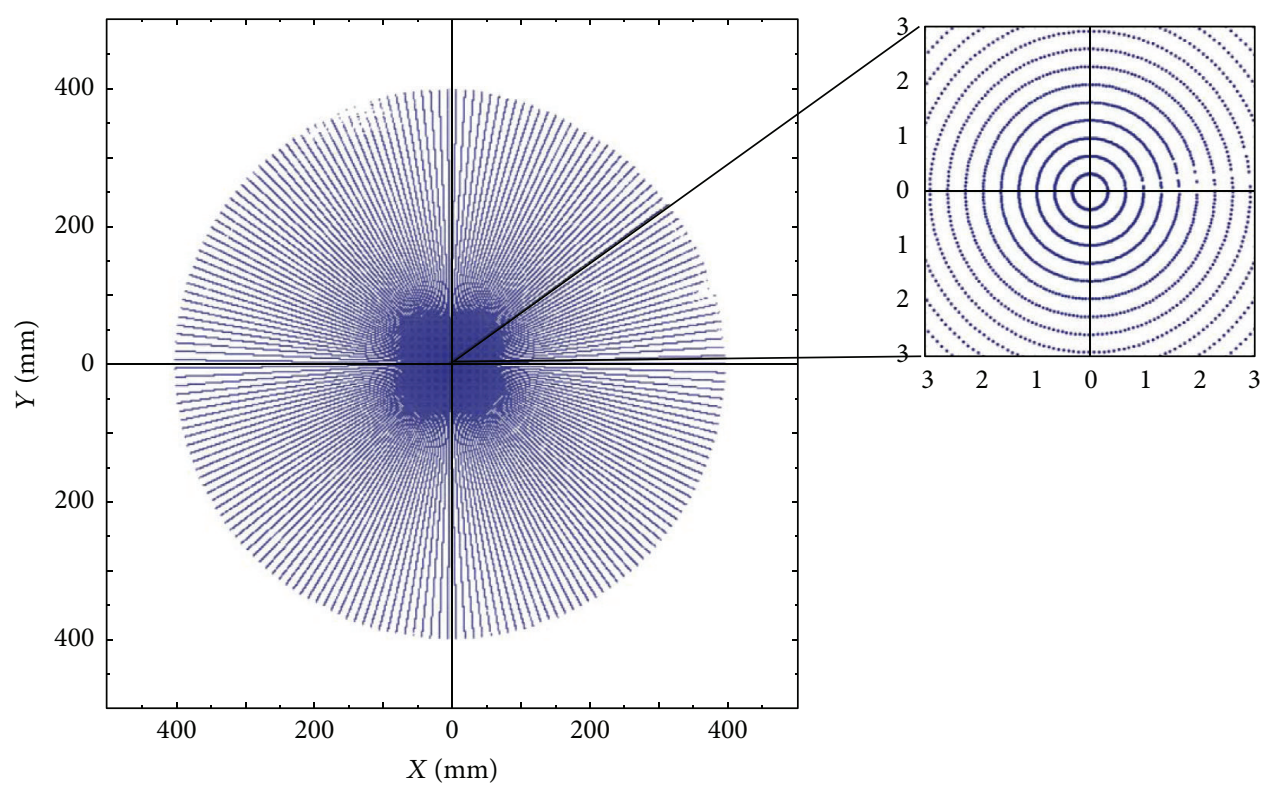

FIGURE 6: Plot of estimation centroids.

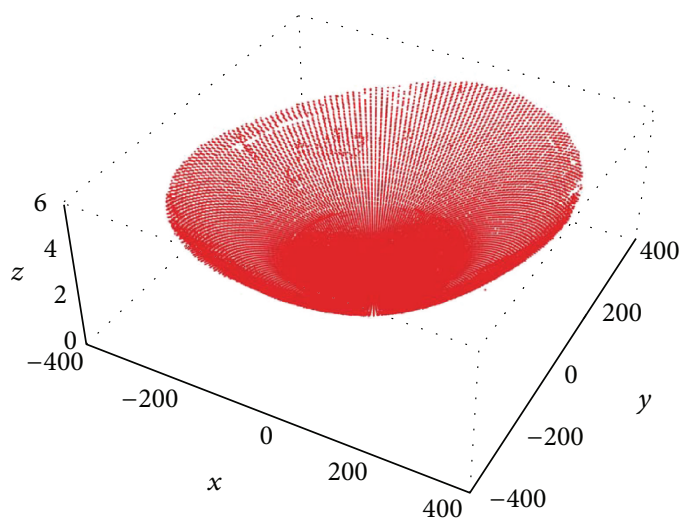

(a)

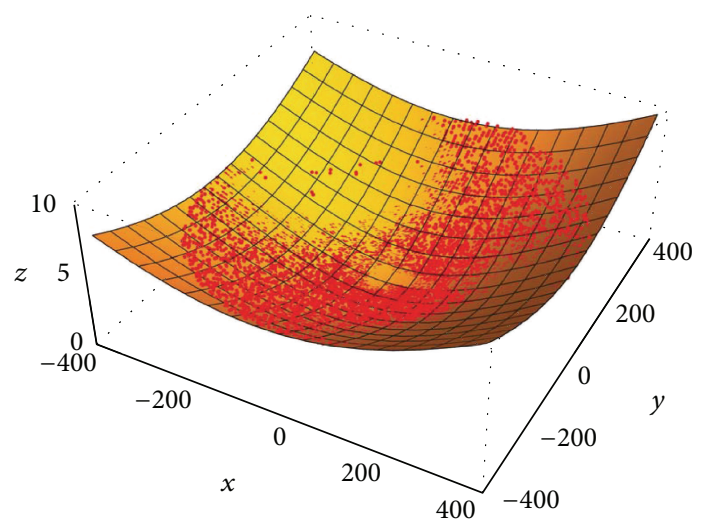

(b)

FIGURE 7: Graphics: (a) reconstruction of the shape of the mirror. (b) The best fit made to the data set.

a Gaussian filter with the "IMFilter" function. Then, the resulting image was binarized to separate the image of the laser line projected and background using the "im2bw" function and an appropriately defined threshold value. Finally, the resulting image centroid estimation was performed for each row of the image.

Having estimated the centroids, displacements were determined with respect to a reference coordinate; in our case, the center point of the sensor is the origin of coordinates for the $X_{i}$ and $Y_{i}$ axes of the image plane. Using (2), we obtained the $Z$ coordinate of the corresponding height at that point.

Coordinates $X$ and $Y$ were obtained by converting the coordinates of the centroids to polar coordinates. The graph in Figure 6 is the set of points in the XY plane. A total of 242,746 points on the surface were evaluated, covering an area of $502,613.27 \mathrm{~mm}^{2}$ on the mirror, testing a $99.9 \%$ of the surface mirror.
4.2. Fitting Data Results. A reconstruction of the threedimensional surface is shown in Figure 7. To determine which surface fits the best, the set of points obtained in the measurements was adjusted with the method of least squares to the equation of a conic surface [15] of the form

$$
\begin{aligned}
z= & \frac{r-\left\{r^{2}-Q\left[\left(x-x_{0}\right)^{2}+\left(y-y_{0}\right)^{2}\right]\right\}^{1 / 2}}{Q}+A x \\
& +B y+z_{0} .
\end{aligned}
$$

Here, $r$ is the paraxial radius of curvature, $Q=k+1$ ( $k$ is the tapering constant of the surface), $x_{0}, y_{0}$, and $z_{0}$ are the coordinates of the vertex, and $A$ and $B$ are the inclination terms of the surface.

Other models for comparing the best fit are made using the paraboloid surface and the spherical surface. The parameters obtained are presented in Table 2. 


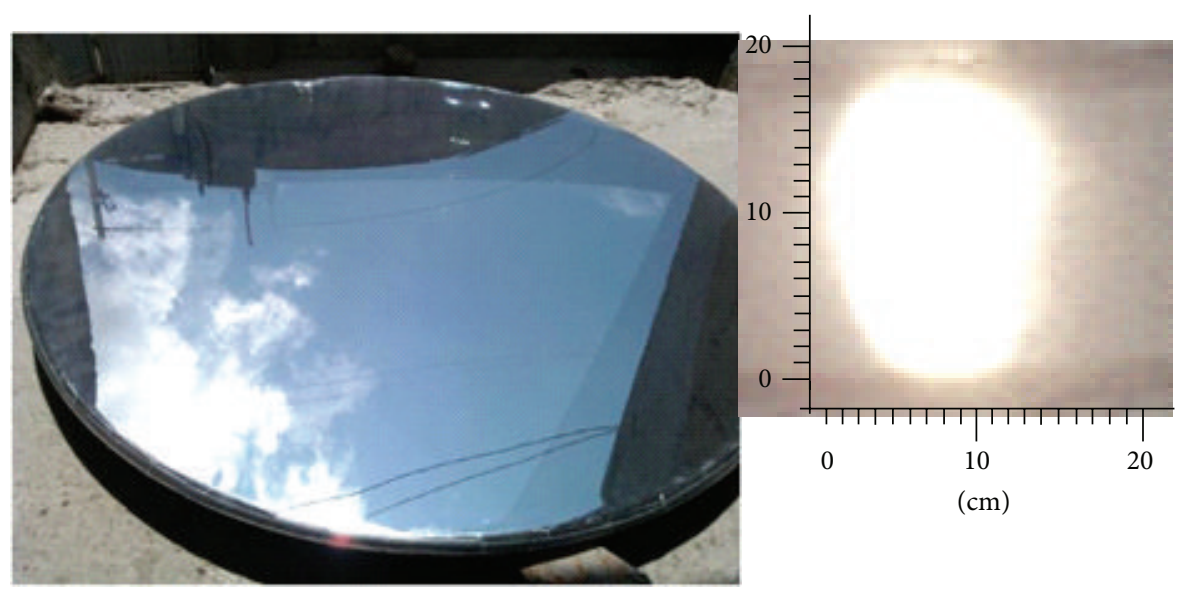

FIGURE 8: Prototype inflatable mirror made of flexible polymeric membranes and the light spot produced by the Sun at the focal plane; reference ruler in $\mathrm{cm}$.

TABle 2: Parameters obtained with the adjustment of the conic, paraboloidal, and spherical surface.

\begin{tabular}{lccc}
\hline Parameters & Conic surface & Paraboloid & Spherical surface \\
\hline$R$ & $10,352.8 \mathrm{~mm}$ & $14,200.1 \mathrm{~mm}$ & $16,402.0 \mathrm{~mm}$ \\
$K$ & -1518.96 & -1.0 & 0.0 \\
$x_{0}$ & $35.7459 \mathrm{~mm}$ & $12.3568 \mathrm{~mm}$ & $-3378.99 \mathrm{~mm}$ \\
$y_{0}$ & $-11.9953 \mathrm{~mm}$ & $212.872 \mathrm{~mm}$ & $-4895.42 \mathrm{~mm}$ \\
$z_{0}$ & $0.283423 \mathrm{~mm}$ & $-1.09116 \mathrm{~mm}$ & $-1116.08 \mathrm{~mm}$ \\
$A$ & $0.00448497 \mathrm{~mm}$ & $0.00305245 \mathrm{~mm}$ & $-0.218892 \mathrm{~mm}$ \\
$B$ & $-0.000899891 \mathrm{~mm}$ & $0.0148914 \mathrm{~mm}$ & $-0.320421 \mathrm{~mm}$ \\
Fit error & $0.151729 \mathrm{~mm}$ & $0.222149 \mathrm{~mm}$ & $0.1569 \mathrm{~mm}$ \\
\hline
\end{tabular}

The results obtained show that the surface that best fits the data set belongs to a conic surface, even though it is pretty close to a spherical surface, Figure 8 . The standard error estimated is of the order of $0.151729 \mathrm{~mm}$, with a standard deviation of the differences between the best fit and the data set of 0.151736 .

\section{Discussion}

In this inflatable mirror made of flexible polymeric membranes both membranes, the reflecting one and the transparent one, were mounted on a metallic ring that keeps both membranes under tension, while being smoothly curved by pneumatic pressure and simultaneously giving structural rigidity to the system. Pneumatic pressure was provided by injecting air at low pressure, allowing the mirror to acquire a lens-like shape. Costs for constructing these $800 \mathrm{~mm}$ diameter mirrors are approximately USD 20.00 each. Durability was tested by leaving inflatable mirror at outdoor conditions for at least 9 months. There were no noticeable changes until the sixth month, when the adhesive of the sealing tape was affected by UV radiation. Once the system was inflated to a given curvature radius, the pressure was kept constant for several days so a minimum air refilling is needed. Controlling the volume of air within the mirrors membranes could help to keep a constant focal distance of the mirror recalling adaptive optics. It should be remarked that the large volume of air contained in between the mirror membranes allows small changes of focal length due to ambient temperature changes and very accurate control of the curvature radius.

We would like to remark on some important features of our mirrors. First, the mirrors are cheap, are easy to manufacture, and are image-forming elements, as can be seen in Figure 9. Nevertheless, they were designed for solar concentration purposes; hence, they easily reach concentration ratio values of 25.1 suns at a $5 \mathrm{~m}$ focal length. They have a reasonably good optical quality, presenting different kinds of aberration. Of course, spherical aberrations are present because the mirror's curvature is acquired by pneumatic pressure (Pascal's law leads to an isotropic stress, which in turn produces spherical surfaces). Another kind of aberration arises because it is a second-surface mirror and the first surface has the curvature radius with opposite sign to the second one. This means that multiple reflections within the first and second surfaces will produce sequentially a set of images at decreasing distances. The last effect is due to small manufacturing defects of the polymeric membranes or during the stretching process when mounting them on the ring edges. This mounting process requires uniform and isotropic stretching of the membrane, which is hard to achieve manually. These nonuniformities give rise to some small wrinkles that, once inflated, will produce borders between sectors of the membrane. Such sectors usually have the same curvature radius, but their optical axes point towards different centers of curvature, producing partially overlapped images or a resulting blurred image of the whole mirror. Furthermore, different stretching coefficients in different directions of the membrane (anisotropic structure of the stretching film) would lead to astigmatic mirrors when inflated. Astigmatism induced by different stretching in two orthogonal directions can be seen in the reconstruction of the surface made by our structured light technique when compared to the best fitting curve shown in Figure 7(b). For the $X Y$-identity straight line, 


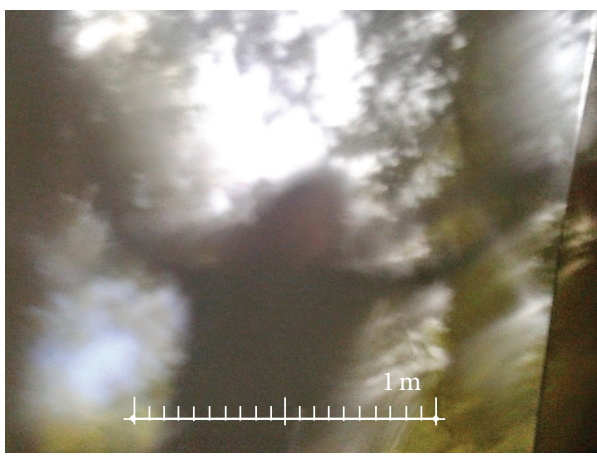

(a)

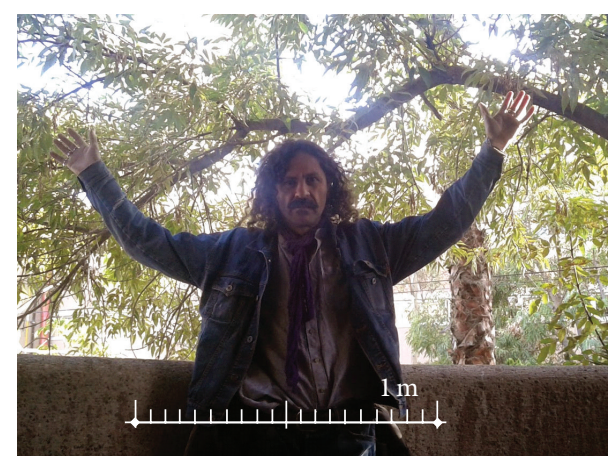

(b)

FIGURE 9: Pictures showing (a) image of one of the authors projected on a white screen, produced by an 800 mm diameter inflatable mirror, with a focal length of $4.0 \mathrm{~m}$. (b) The same scene shot directly.

most of the measured $Z$ coordinates of the measured surface lay over the best fitting surface, while for the orthogonal direction most of the measured heights lay below the fitting surface. This astigmatism was corroborated by the elongated shape of the Sun's image shown in Figure 9 resulting from two different focal lengths, corresponding to two different curvature radii of the mirror surface along two directions.

All these detrimental features can be worked systematically in order to correct each aberration if the mirror is intended for higher quality systems, such as solar furnaces or even telescopes.

A thermal-performance test of a single-mirror system and a naked receiver shows an efficiency of 37\% for lowtemperature applications, such as domestic water heating, food industry, or laundry processes, which represent a broad niche market, not yet fully covered (global reference: 68.7 $\mathrm{Mm}^{2}$ [16], local reference: $2.3 \mathrm{Mm}^{2}$ [17], both on solar thermal collector). In other feasible applications of fluid heating by solar power, as well as applications in sterilization, pasteurization, drying, hydrolyzed, cleaning, polymerization, distillation, and evaporation [18], inflatable mirrors represent a promising approach as well. Even though these mirrors are second-surface reflectors, their performance for concentration purposes is very good and helps to reduce the size and weight of the otherwise thick supporting rings, if negative pressure is intended to be used in order to produce first surface mirrors.

Yet, using an array of seven mirrors hexagonally arranged allowed us to reach concentration factors of 87 suns, as shown in Figure 10, keeping costs below USD 200.00 and weights under $15 \mathrm{~kg}$. This paves the way to high-temperature (higher concentration ratios) applications, such as Stirling engine-parabolic dish or Rankine cycle electricity generation schemes [13], as well as using high-efficiency refrigerated photovoltaic cells under concentrated solar radiation.

\section{Conclusions}

Cheap, good optical quality inflatable membrane mirror was built. Surface shape characterization was done using

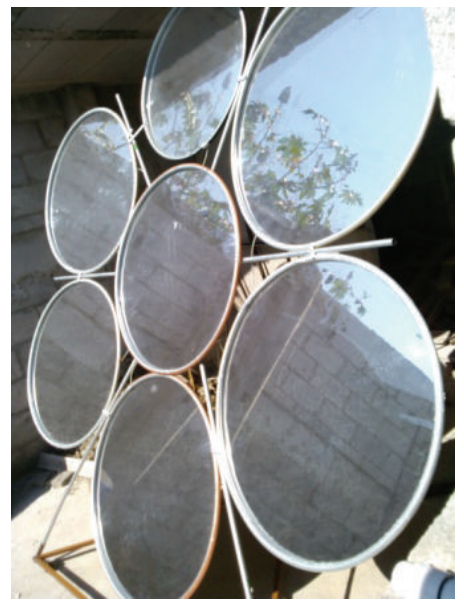

Figure 10: Prototype, hexagonal distribution array using seven inflatable membranes mirror.

structured light techniques with resolution better than $1 \mathrm{~mm}$. Fitting measurements to a conical curve gives parameter values corresponding to spherical-sector mirrors, instead of a Hencky surface (oblate spheroid sector). Concentration ratios of 25 suns are easily achieved with a single mirror, while, using a sevenfold array, 87 suns are reached. Thermal efficiency of a simple system of one mirror and a naked receiver was determined as being higher than $37.51 \%$ in spite of being used in second-surface configuration. Manufacturing costs and large durability of these light mirrors considered together with their performance and energy costs from fossil fuels (including natural gas) make inflatable mirrors promising in applications ranging from domestic water heating to Stirling-parabolic dish electricity production, if faceted arrays of these light weighted mirrors are used.

\section{Conflict of Interests}

The authors declare no conflict of interests. 


\section{Authors' Contribution}

Felipe Patiño-Jiménez, Yuri Nahmad-Molinari, and Víctor Iván Moreno-Oliva contributed in designing, measuring, discussing, and writing down the paper, while Fátima De Los Santos-García and Agustin Santiago-Alvarado contributed in construction and characterization.

\section{Acknowledgments}

Felipe Patiño-Jiménez acknowledges support from Conacyt through a Scholarship no. 317090 and Queretaro Technological University through job support. Yuri Nahmad-Molinari acknowledges support through Project IDB-ATN/KK-11514RG and Conacyt 221961.

\section{References}

[1] A. B. Meinel and M. P. Meinel, Inflatable Membrane Mirrors for Optical Passband Imagery, Jet Propulsion Laboratory California Institute of Technology, Pasadena, Calif, USA, 2000.

[2] M. Soh, J. H. Lee, and S.-K. Youn, "Optimal thickness variation of an inflatable circular membrane mirror," Journal of the Korean Physical Society, vol. 44, no. 4, pp. 854-858, 2004.

[3] Módulo: Energía Solar Termoeléctrica, March 2014, http://api .eoi.es/api_vl_dev.php/fedora/asset/eoi:45321/componente45319 .pdf.

[4] J. K. Bishop and W. B. Rossow, "Spatial and temporal variability of global surface solar irradiance," Journal of Geophysical Research C, vol. 96, no. 9, pp. 16839-16858, 1991.

[5] Total Primary Energy Supply, March 2014, http://www.ez2c.de/ $\mathrm{ml} /$ solar_land_area/index.html.

[6] V. Moreno-Oliva, E. Román-Hernández, S. Rafael-Esesarte, and R. García-Hernández, "Prueba de calidad en la superficie reflectora de un concentrador solar de canal parabólico con el uso de luz estructurada," CienciaUAT, vol. 8, no. 2, pp. 68-72, 2014, http://www.revistaciencia.uat.edu.mx/index.php/CienciaUAT/article/view/296/159.

[7] M. Wang and K. Siddiqui, "The impact of geometrical parameters on the thermal performance of a solar receiver of dish-type concentrated solar energy system," Renewable Energy, vol. 35, no. 11, pp. 2501-2513, 2010.

[8] K. Lovegrove, G. Burgess, and J. Pye, "A new $500 \mathrm{~m}^{2}$ paraboloidal dish solar concentrator," Solar Energy, vol. 85, no. 4, pp. 620-626, 2011.

[9] E. Kussul, T. Baidyk, F. Lara-Rosano, J. M. Saniger, N. Bruce, and C. Estrada, "Micro-facet solar concentrator," International Journal of Sustainable Energy, vol. 27, no. 2, pp. 61-71, 2008.

[10] J. Gene, Desing of a low cost solar concentrator [Mechanic Engineer Master Degree], Texas Technology University, 1986.

[11] Z. Liu, J. Lapp, and W. Lipiński, "Optical design of a flat-facet solar concentrator," Solar Energy, vol. 86, no. 6, pp. 1962-1966, 2012.

[12] G. Zanganeh, R. Bader, A. Pedretti, M. Pedretti, and A. Steinfeld, "A solar dish concentrator based on ellipsoidal polyester membrane facets," Solar Energy, vol. 86, no. 1, pp. 40-47, 2012.

[13] G. Grossman and G. Williams, "Inflatable concentrator for solar propulsion and dynamic space power," Transactions of the ASME-Journal of Solar Energy Engineering, vol. 112, no. 4, pp. 229-236, 1990.
[14] T. Wendelin, A. Lewandowski, and A. Dobos, "Software SolTrace," General-purpose ray trace code developed by the National Renewable Energy Laboratory (NREL) to model solar power optical systems and analyze their performance, Version 2012.7.9, http://www.nrel.gov/csp/soltrace/download.html.

[15] D. Malacara, Optical Shop Testing, Appendix 1: An Optical Surface and Its Characteristics, Wiley, New York, NY, USA, 2nd edition, 1992.

[16] German Energy Agency, 2014, http://www.renewables-madein-germany.com/es.html.

[17] Sector de Energías Renovables en México, SENER, CRE y CFE, 2014, http://mim.promexico.gob.mx/wb/mim/energias_perfil_ del_sector.

[18] S. Kalogirou, Solar Energy Engineering: Processes and Systems, 1st edition, 2009. 

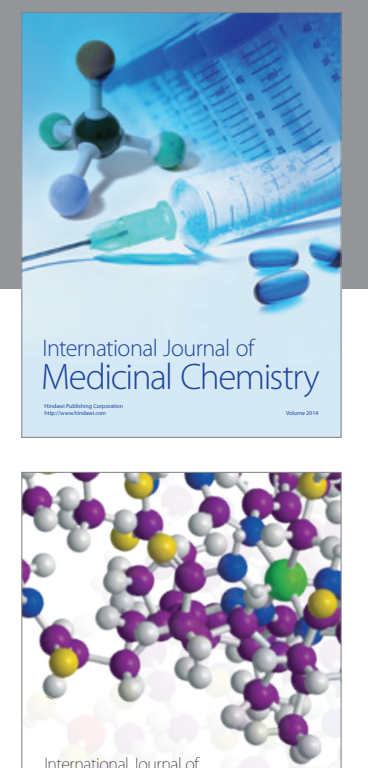

\section{Carbohydrate} Chemistry

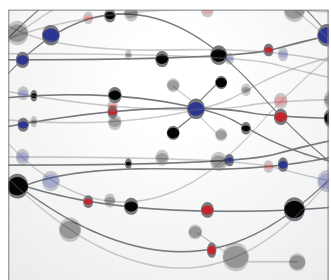

The Scientific World Journal
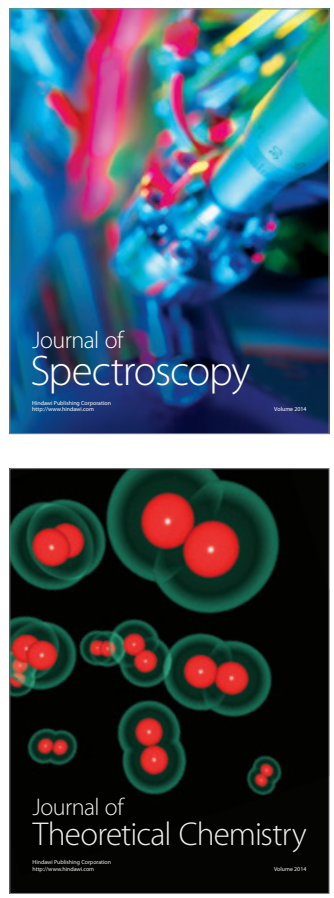
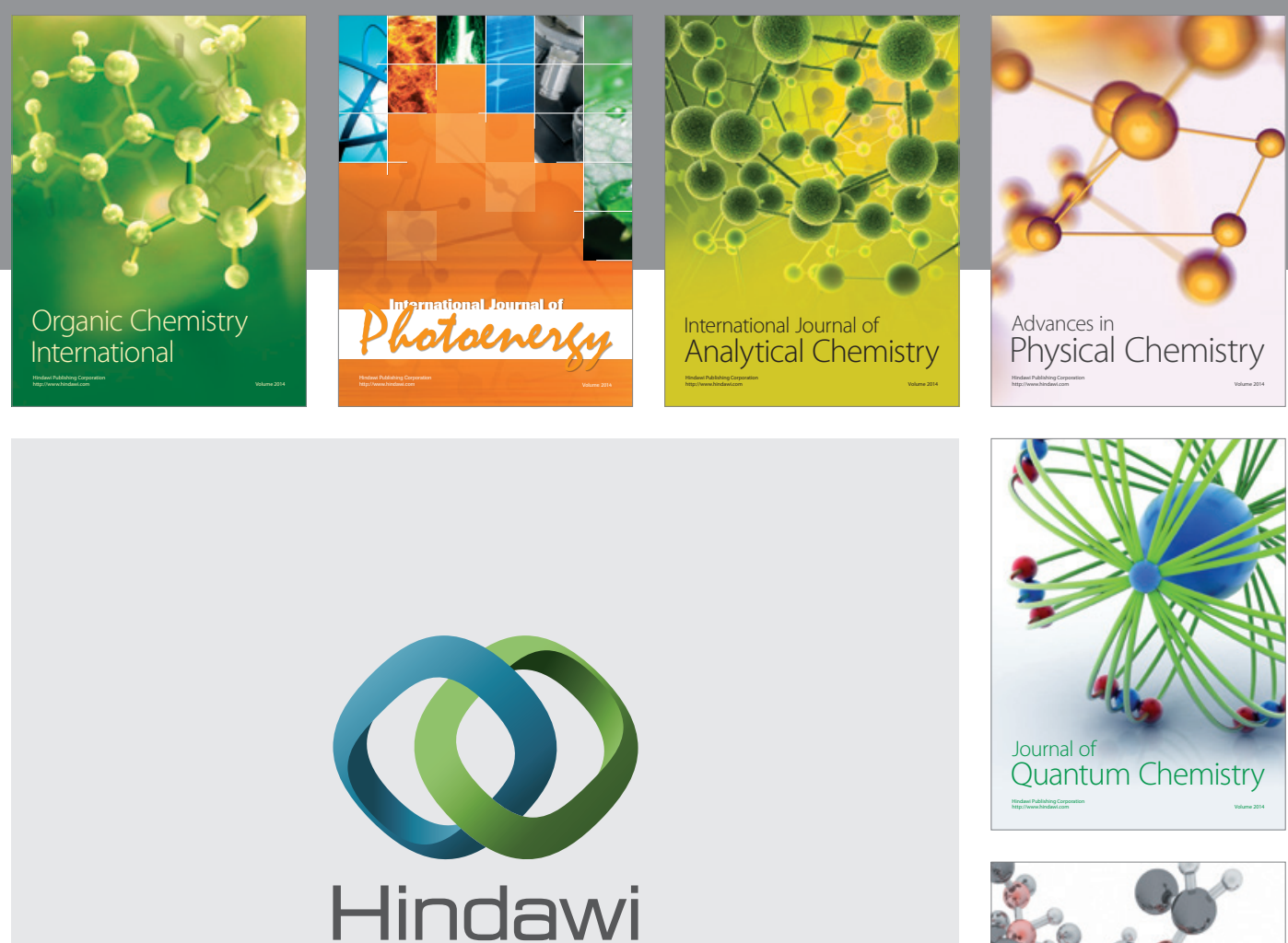

Submit your manuscripts at

http://www.hindawi.com

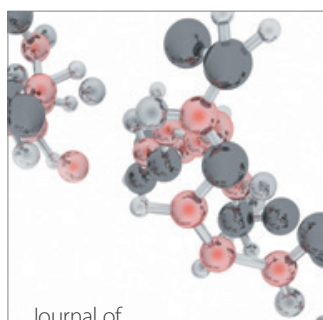

Analytical Methods

in Chemistry

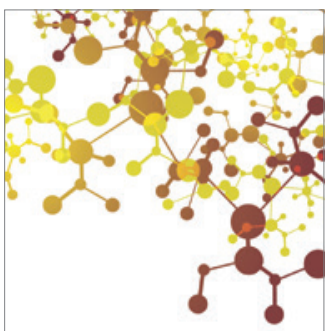

Journal of

Applied Chemistry

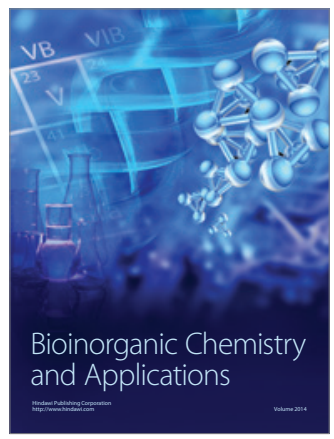

Inorganic Chemistry
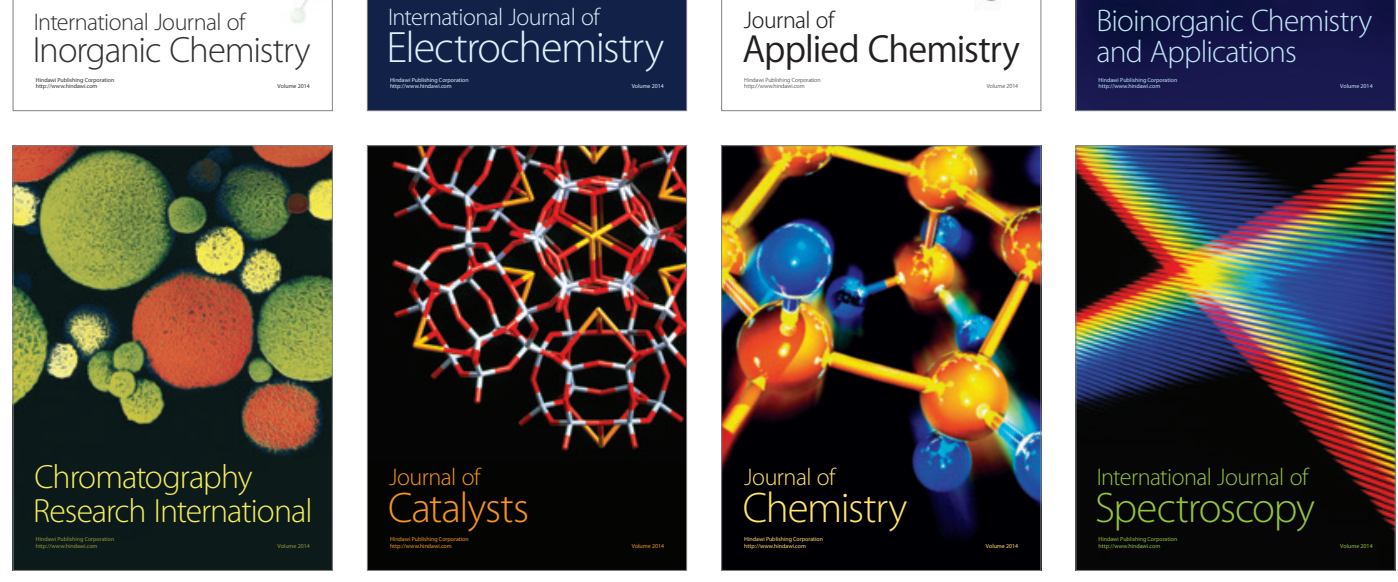